\title{
A Functional Model of Limbic System of Brain
}

\author{
Takashi Kuremoto ${ }^{1}$, Tomonori Ohta ${ }^{1}$, Kunikazu Kobayashi ${ }^{1}$, Masanao Obayashi ${ }^{1}$, \\ ${ }^{1}$ Graduate School of Science and Engineering, Yamaguchi University, \\ 755-8611 Tokiwadai 2-16-1, Ube, Japan \\ \{wu, koba, m.obayas\}@yamaguchi-u.ac.jp
}

\begin{abstract}
A functional model of limbic system of brain is proposed by combining four conventional models: a chaotic neural network (CNN), a multilayered chaotic neural network (MCNN), a hippocampus-neocortex model and an emotional model of amygdala. The composite model can realize mutual association of multiple time series patterns and transform short-term memory to long-term memory. The simulation results showed the effectiveness of the proposed model, and this study suggests the possibility of the brain model construction by means of integration of different kinds of artificial neural networks.
\end{abstract}

\section{Introduction}

Artificial neural networks have been developed for decades from last century and successfully used in fields of function approximation, optimization, pattern recognition, intelligent control, and so on. Functionally, Hopfield network, a recurrent neural network in which all connections are symmetric, has the ability of stationary auto-association, meanwhile chaotic neural networks can realize dynamic association $[1,2]$. Emotion models are also proposed and applied in control systems recently [3]. However, realization of an integrated artificial brain model including multiple functions of brain is still a high hurdle even though learning models, memory models, emotion models and other functional neural networks have been proposed.

The limbic system of mammalian brain locates on the under of brain including the parts of hippocampus, amygdala, anterior thalamic nuclei, and entorhinal cortex. It serves to a variety of functions including to transform short-term memory (working memory) into long-term memory (declarative memory), to control the emotional response and support decision of behaviors. In this paper, we propose a limbic system mathematical model, which combines several functional models, to present how input patterns are stored in hippocampus and transformed into long-term memory on cortex, and how emotion models participate in these processes. Our functional model of the limbic system is developed according to the following points:

1) Hippocampus plays an important role of transforming intermediate-term memory to long-term memory. Ito et al proposed a hippocampus-cortex model [4]

A part of this study was supported by JSPS-KAKENHI (No.18500230, No.20500277 and No.20500207). 
and a hippocampus-neocortex model [5] which composed a circuit of cortex - dentate gyrus - hippocampus - cortex to realize episode memory and long-term memory, respectively. For its characteristic structure of neuroanatomy and computational availability of mathematics, we adopt this hippocampus-cortex model as a part of our model of limbic system.

2) The structure of hippocampus is organized with stratified slices and chaotic response is observed appearing among these neurons. We proposed a new hippocampus-neocortex model to realize mutual association and long-term memory in our previous works $[8,9]$. The main difference between our model and Ito et al's model is that a model of CA3 layer of hippocampus was presented by multi-layered chaotic neural networks (MCNN) [7]. MCNN is also used to realize dynamical memory process in this study.

3) Amygdala plays important roles in emotional responses of brain, and neighbored to hippocampus in location. We consider that emotion can promote the efficiency of memory process, so adopt an emotion model proposed by Balkenius and Moren [3] [13] into our model of limbic system. The main components of the emotion model are thalamus, amygdala, orbitofrontal cortex and sensory cortex. The model has been used successfully in an intelligent control system [14] and we also used it to improve the chaos control process in hippocampus-neocortex model efficiently [10].

According to the knowledge of neuroanatomy, neurophysiology and physiological psychology, we propose a higher function model of limbic system fusing hippocampus-neocortex model, MCNN and emotion model described above in this paper.

\section{A Model of Limbic System}

A model of limbic system which is composed with a hippocampus-neocortex model $[8,9]$ and an amygdala model [3] [13] is shown in Fig. 1. The hippocampus-neocortex model consists of a memory circuit including cortex - dentate gyrus - hippocampus cortex given by Ito et al [4] [5]. Entorhinal cortex and CA2 of hippocampus are omitted for their weak connections. A chaotic model of CA3 layer in hippocampus is adopted in with multi-layered chaotic neural networks (MCNN) [7] which combines two chaotic neural networks [1] instead of the single layer of Ito et al's model to serve intermediate-memory processing. MCNN showed its effectiveness of mutual association of plural time series patterns and long-term memory formation in our previous simulations [7] [8] [9]. However, emotional effect is not considered in the memorization process of MCNN, likewise almost of conventional association systems. In fact, amygdala, which locates adjacent of hippocampus, plays important roles in the emotional responses such as fear and aggression, and also in the memorization process such as learning of Pavlovian fear conditioning and enhancing of long-term memory formation [14]. We adopt an amygdala model given by Balkenius and Moren [3] [13] here to evaluate and promote the efficiency of memory processing. 


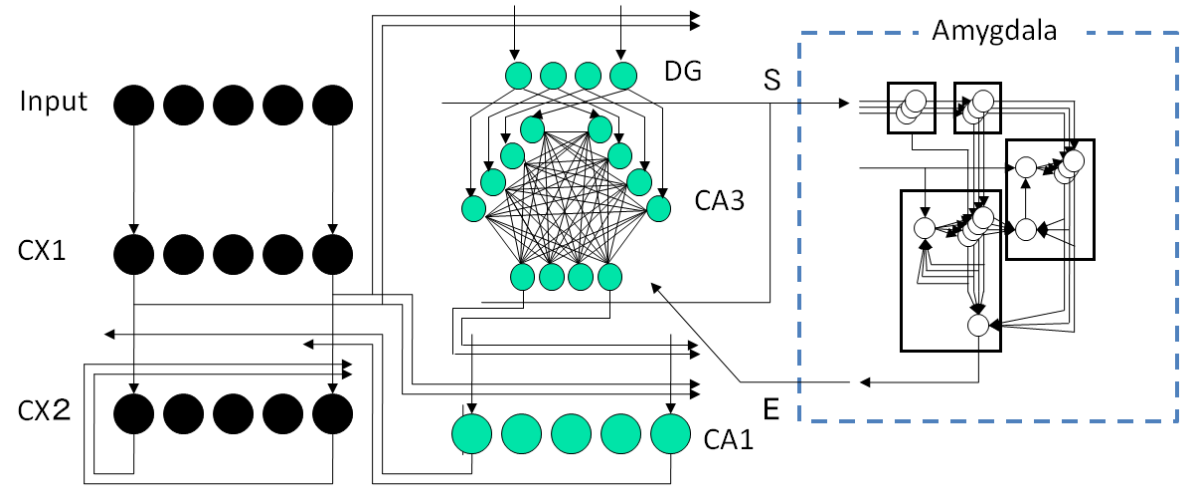

Fig. 1. A model of limbic system proposed in this paper. Neurons in cortex, hippocampus and amygdala are expressed with dark, gray and white dots respectively. Signals are expressed with arrow lines.

\subsection{A hippocampus-neocortex Model}

The dark dots and gray dots in Fig. 1 express neurons of neocortex and hippocampus, and symbols of CX1, CX2, DG, CA3 and CA1 denote the first layer of cortex, the second layer of cortex, dentate gyrus, the third layer of hippocampus and the first layer of hippocampus respectively. The signal flow of the hippocampus-neocortex model is showed with arrows, i.e.: input stimuli (Input layer) $\rightarrow$ sensory memory $(\mathrm{CX} 1) \rightarrow$ short-term memory (CX2) $\rightarrow$ intermediate memory (DG) $\rightarrow$ Hebbian learning and chaotic processing of storage and recollection (CA3) $\rightarrow$ decoding (CA1) $\rightarrow$ long-term memory (CX2). The output of CA3, which is a result of chaotic memory processing, projects to Amygdala and the output of Amygdala is input to CA3 to realize chaotic state control of MCNN in CA3.

The dynamics of association cortex is given as following.

$$
\begin{gathered}
I_{i}=\left\{\begin{array}{l}
1 \cdots \text { excitatory } \\
0 \cdots \text { inhibitory }
\end{array}\right. \\
x_{i}^{c x 1}(t)=I_{i}(t) . \\
x_{i}^{c x 2}(t)=f\left(\sum_{j=0}^{N} w_{i j}^{c x 2 \cdot c x 2} x_{j}^{c x 2}(t-1)+\right. \\
\left.w^{c x 2 \cdot c x 1} x_{i}^{c x 1}(t)+w^{c x 2 \cdot c a 1} x_{i}^{c a 1}(t)-\theta^{c x}\right) .
\end{gathered}
$$


Where $I_{i}(t)$ is the output value of the $i$ th neuron in the Input layer of association cortex, $x_{i}^{c x 1}(t)$ and $x_{i}^{c x 2}(t)$ are the output value of the $i$ th neuron in CX1 and $\mathrm{CX} 2$ respectively, $w_{i j}^{c x 2 \cdot c x 2}$ denotes the weight of connection (variable) between the $j$ th neuron (output) and the $i$ th neuron in the CX2 layer, $w^{c x 2 \cdot c x 1}$ $w^{c x 2 \cdot c a 1}$ denote the weights of connections (fixed) between layers of CX1 and CX2, CX2 and CA1 respectively, $x_{i}^{c a 1}(t)$ is the output of the $i$ th neuron in CA1, $\theta^{c x}$ is a threshold value of cortex neurons, $N$ is the number of neurons in CX1 as same as CX2, $f$ expresses a step function. Eq. (3) endows CX2 associative function for input patterns and long-term memory formation function for output patterns of hippocampus.

The learning rule of the synapses in CX2 is given by Eq. (4) which is a Habbian rule using the different output of neurons in time $t$ and $t-1$.

$$
\Delta w_{i j}^{c x 2 \cdot c x 2}=\alpha_{h c} \cdot x_{i}^{c x 2}(t) x_{j}^{c x 2}(t-1) .
$$

Where $\alpha_{h c}$ is a parameter of learning rate.

Hippocampus composes DG, MCNN and CA1 neurons. DG executes pattern encoding (Eq. (5)) with a competition learning (Eq. (6)).

$$
\begin{gathered}
x_{i}^{d g}(t)=\left\{\begin{array}{l}
\text { random } \subset(0,1) \\
f\left(\sum_{j=0}^{N} w_{i j}^{d g \cdot c x 1} x_{j}^{c x 1}(t)-\theta^{d g}\right) \cdots \quad(\text { generally })
\end{array} .\right. \\
\Delta w_{i j}^{d g \cdot c x 1}=\beta_{h c} \cdot x_{i}^{d g}(t) x_{j}^{c x 1}(t) .
\end{gathered}
$$

Where $w_{i j}^{d g \cdot c x 1}$ denotes the weight of connection between the $i$ th neuron in CX1 (output) and the $j$ th neuron in DG, $x_{j}^{c x 1}$ is the output of the $i$ th neuron in CX1, $\theta^{d g}$ is a threshold value of DG neurons, $\beta_{h c}$ is a learning rate and $\alpha_{h c}<\beta_{h c}$.

CA3 accepts the encoded information from DG and executes chaotic processing of storage and recollection with MCNN. It consists of two CNN layers which dynamics is given by Eq. (12) - Eq. (15) and one output layer which neuron's output is given by Eq. (7) - Eq. (9).

$$
k=\arg \max _{i} \sum_{j=0}^{n} w_{i j}^{\text {ca3outccnn } 1}\left(2 x_{j}^{c n n 1}(t)-1\right) .
$$




$$
\begin{aligned}
& k=\arg \max _{i} \sum_{j=0}^{n} w_{i j}^{\text {ca } 3 \text { out cnn } 2}\left(2 x_{j}^{\text {cnn } 2}(t)-1\right) . \\
& x_{i}^{\text {ca3out }}(t)=\left\{\begin{array}{lll}
1 & \cdots & (i=k) \\
0 & \cdots & (i \neq k)
\end{array} .\right.
\end{aligned}
$$

Here the $j$ th neuron in $\mathrm{CNN} 1 x^{c n n 1}{ }_{j}(t)$ and the $j$ th neuron in CNN2 $x^{c n n 2}{ }_{j}(t)$ are used to transform the output of MCNN by the $i$ th neuron in output layer of MCNN $x_{i}^{\text {c3out }}(t) w^{\text {ca3out.cnn }{ }_{i j}}$ and $w^{\text {ca3out.cnn } 2}{ }_{i j}$ denote the connections between the output layer and CNN1, CNN2 respectively. Learning rule is given as $\Delta w_{i j}^{\text {ca3out } \cdot c n n}=\frac{1}{n} x_{i}^{\text {ca3out }}(t) x_{j}^{c}{ }^{n}\left(t^{\prime}\right)$.

Mutual associative memory process is realized by the output layer of MCNN receives the output of $\mathrm{CNN} 1$ and $\mathrm{CNN} 2$, i.e., Eq. (7) and Eq. (8), alternatively. In fact, when there are two time series patterns are processed by the two CNN layers of MCNN, one layer of CNN plays a role of static external stimuli meanwhile another layer of $\mathrm{CNN}$ executes chaotic storage or recollection processing dynamically. In another word, CNN layers fire alternatively in CA3. To switch their roles of CNNs, we used a simple threshold in [7] [8] [9], however, an emotional control given by subsection 2.2 and subsection 2.3 can raise the performance of MCNN [10].

CA1 in hippocampus decodes output pattern of MCNN which is expressed by $n$ neurons to patterns stored by associative cortex CX2 which has $N$ neurons (Eq. (10)). Hebbian learning rule is described by Eq. (11) where input from CX1 is a teacher $\operatorname{signal}\left(w_{i j}^{c a 1 \cdot c x 1}=1.0\right)$.

$$
\begin{gathered}
x_{i}^{c a 1}(t)=f\left(\sum_{j=0}^{n} w_{i j}^{c a 1 \cdot c a 3} x_{j}^{c a 3 o u t}(t-1)+\right. \\
\left.w^{c a 1 \cdot c x 1} x_{i}^{c x 1}(t)-\theta^{c a 1}\right) . \\
\Delta w_{i j}^{c a 1 \cdot c a 3}=\beta_{h c} \cdot x_{i}^{c a 1}(t) x_{j}^{c a 3 o u t}(t) .
\end{gathered}
$$

Where $\beta_{h c}$ is a parameter of learning rate, $i=1,2, \ldots, N$.

CNN1 and CNN2 are Adachi \& Aihara's CNN proposed in [1] [2] and combined to each other in our MCNN model [7]. The dynamics are described by Eq. (12) - Eq. (15).

$$
\begin{gathered}
x_{i}(t+1)=g\left(y_{i}(t+1)+z_{i}(t+1)+\gamma \cdot v_{i}(t+1)\right) . \\
y_{i}(t+1)=k_{r} y_{i}(t)-\alpha x_{i}(t)+a_{i} .
\end{gathered}
$$




$$
\begin{aligned}
& z_{i}(t+1)=k_{f} z_{i}(t)+\sum_{j=1}^{n} w_{i j} x_{j}(t) . \\
& v_{i}(t+1)=k_{e} v_{i}(t)+\sum_{j=1}^{n} W_{i j}^{*} x_{j}^{*}(t) .
\end{aligned}
$$

Where $x_{i}(t)$ is the output value of $i$ th neuron at time $t, n$ is the number of neurons of input layer, $w_{i j}$ is the weight of connection from $j$ th neuron to $i$ th neuron, $y_{i}(t)$ denotes internal state of $i$ th neuron, $\alpha$ is a learning rate of $i$ th neuron, $k_{f}, k_{r}, k_{e}$ are damping rates, $a_{i}$ is a parameter as the summation of threshold and external input, $\gamma$ is a rate of effectiveness from another layer, $W^{*}{ }_{i j}$ denotes the weight of connection from $j$ th neuron of different CNN layer to $i$ th neuron in current CNN layer, $x_{j}{ }_{j}(t)$ is the output value of $j$ th neuron in different $\mathrm{CNN}$ layer at time $t . g(\cdot)$ is a sigmoid function.

When a MCNN is composed by two CNN layers, learning rules of the connections between the CNN layers $W^{*}{ }_{i j}$ are described by Eq. (16) and Eq. (17).

$$
\begin{aligned}
\Delta W_{i j}^{c n n 1 \cdot c n n 2} & =\beta \cdot x_{i}^{c n n 1}(t) x_{j}^{c n n 2}(t) . \\
\Delta W_{i j}^{c n n 2 \cdot c n n 1} & =\beta \cdot x_{i}^{c n n 2}(t) x_{j}^{c n n 1}(t) .
\end{aligned}
$$

Where $\beta$ is a parameter of learning rate, usually $\beta=1 / m, m$ is the number of stored patterns.

Conventionally, we calculated the temporal change of the internal state of a CNN layer $\triangle x(t)$, and when $\triangle x(t)$ is less than a threshold $\theta$, the chaotic retrieval of the layer is stopped by changing values of parameters $k_{r}, k_{f}$ into zero, as a result, the CNN layer becomes to a Hopfield model. The recalled pattern of one CNN layer provides an input pattern to the other CNN layer, and the network realizes mutual association and one-to-many retrieval for plural time series patterns [7] [8] [9].

\subsection{An Amygdala-Hippocampus Model}

Balkenius \& Moren's computational amygdala model [3] [13], which is shown in the right part of limbic system model in Fig. 1, is combined with hippocampus-neocortex described above to evaluate and promote the performance of memory processing. Recently, we proposed an amygdala-hippocampus model which showed faster storage ability and higher precision of recollection of plural time series patterns dynamical association [10]. The main idea of the adoption of emotional model comes from the consideration that unstable state of hippocampus may result emotional response, i.e., arousal of amygdala, or the high value of amygdale output may enhance memory processing happened in hippocampus.

The dynamics of the amygdale model is described as follows: 


$$
\begin{gathered}
A_{i}=V_{i} S_{i} . \\
O_{i}=W_{i} S_{i} . \\
E=\sum_{i} A_{i}-\sum_{i} O_{i} . \\
\Delta V_{i}=\alpha_{A M Y}\left(S_{i} \max \left(0, R-\sum_{j} A_{j}\right) .\right. \\
\Delta W_{i}=\beta_{A M Y}\left(S_{i} \sum_{j}\left(O_{j}-R\right)\right) .
\end{gathered}
$$

Where $S_{i}$ denotes input stimuli from sensory cortex and thalamus to $i$ th neuron in amygdala, $A_{i}$ denotes the output of $i$ th neuron in amygdala, $O_{i}$ denotes the output of $i$ th neuron in orbitofrontal cortex, $E$ is the output of amygdala which control the internal state of MCNN by tuning damping parameters as same as our conventional method. $\alpha_{A M Y}, \beta_{A M Y}$ are learning rates, $V_{i}, W_{i}$ are variables of connections in Amygdala model. Reward $R$ coming from other area of brain is used to renew Eq. (21) and Eq. (22) which belongs to reinforcement learning algorithm.

Suppose that the unstable degree of a CNN provides a reward $R$ to amygdala model, then an emotional control can be realized by Eq. (18) - Eq. (27).

$$
S_{i}=\frac{\sum_{j=i^{*} \Delta N}^{\Delta N^{*}(i+1)}\left(x_{j}(t+1)-x_{j}(t)\right)}{\Delta N}, \quad, \quad \text { where } i=0,1, \cdots, N_{A M Y}-1 .
$$

$$
\Delta \mathrm{N}=\frac{n}{N_{A M Y}}
$$

$$
\begin{gathered}
R=\left\{\begin{array}{lll}
1.0 & \cdots & \left(F>n_{R}\right) \\
0.0 & \cdots & (\text { else })
\end{array} .\right. \\
F=\frac{\sum_{i=0}^{N_{A M Y}} g\left(S_{i}-\theta_{R}\right)}{N_{A M Y}} .
\end{gathered}
$$

$$
\text { CNN state: }\left\{\begin{array}{llc}
\text { chaotic } & \cdots & \left(E>\theta_{A M Y}\right) \\
\text { non-chaotic } & \cdots & (\text { else })
\end{array}\right. \text {. }
$$


Where $S_{i}$ in Eq. (23) corresponds to input of Amygdala (Eq. (19) and Eq. (20)), $x_{j}$ denotes the output of chaotic neuron in CNN layers, $N_{A M Y}$ is the number of amygdala layer in Amygdala model, $\mathrm{n}$ is the number of CNN layers, $R$ is a reward according to Eq. (25) where $F$ expresses fire rate of the neurons in amygdala layer, $\theta_{R}$ is a threshold of the output of the amygdala layer, $n_{R}$ is a threshold of the reward function Eq. (25), $g(\cdot)$ is a Sigmoid function. The output of Amygadala $E_{\text {controls }}$ state of MCNN with threshold $\theta_{A M Y}$ which is described in Eq. (27).

\section{Simulations}

To confirm the effectiveness of the proposed model of limbic system, we performed two kinds of simulations using a personal computer loaded a Pentium 4 CPU. The fist simulation was designed to compare the mutual association abilities between conventional MCNN and Amygdala - Hippocampus model. This simulation was also described in [10], however, new statistical results would be reported more in this paper. The second simulation was designed to compare one-to-many association abilities, and long-term memory formation abilities between Amydala-Hippocampus model and the model of limbic system proposed in this paper.

\subsection{Simulation of Mutual Association}

Two time series patterns used in mutual association simulation of MCNN and Amygdala-Hippocampus model are as same as those in [7] and [10]. All parameters value or their initial value used in these simulations were decided by empirical knowledge or according to the previous works and they are shown in Table 1. The comparison of storage time and recollection time of different models is shown by Table 2. Amygdala-Hippocampus model had a faster storage than MCNN however a slower recollection. The results can be explained as that emotional model enhanced the storage processing in the meaning of efficient and enhanced the recollection more "carefully" in the meaning of precision. In fact we confirmed that retrieved patterns using emotional control method showed their completeness meanwhile MCNN failed sometime. Illustrations of the results by MCNN are omitted here for want of space, and results by Amygadala-Hippocampus model are shown in Fig. 2.

\subsection{Simulation of Long-term Memory}

Two time series patterns shown in Input layer of Fig. 3 similar to the simulations of Ito et al's [5] were used to investigate the association and long-term memory formation abilities of proposed model of limbic system. Binary patterns in each time series were orthogonal, and a 4-step interval between the two time series was set to distinguish them. The procedure of simulation is described according to time sequence as following: 
A. Input the two time series patterns. The first pattern of each time series was same to serve as a key pattern of one-to-many association.

B. Intermediate memory recollection. Input the key pattern to associate time series patterns stored on the time A.

C. Recollection without hippocampus. Stop the output of hippocampus and amygdala temporarily to investigate long-term memory formation on CX2.

D. Consolidate long-term memory with a long-term potentiation (LPT) process, i.e., input the key pattern repeatedly. Hippocampus works to transform intermediate memory to long-term memory.

E. Recollection of long-term memory. Stop hippocampus and amygdala to investigate long-term memory formation on CX2.

Table 1. Parameter values (or initial values) used in the simulations.

\begin{tabular}{cll}
\hline Symbol & \multicolumn{1}{c}{ Description } & \multicolumn{1}{c}{ Value } \\
\hline$N$ & Number of neurons in association cortex & 50 \\
$n$ & Number of neurons in association cortex & 30 \\
$w_{i j}^{c x 2 c c x 1}$ & Weight of connection from CX1 to CX2 & 1.0 \\
$w_{i j}^{c x 2 c c a 1}$ & Weight of connection from CX2 to CA1 & 1.0 \\
$\alpha_{h c}$ & Learning rate in association cortex & 0.0015 \\
$\beta_{h c}$ & Learning rate in hippocampus & 1.0 \\
$\alpha_{A M Y}$ & Learning rate in amygdala part & 0.2 \\
$\beta_{A M Y}$ & Learning rate in ortbitofrontal part & 0.8 \\
$k_{f}$ & Damping coefficient in CNN & 0.02 \\
$k_{e}$ & Damping coefficient in CNN & 0.02 \\
$k_{r}$ & Damping coefficient in CNN & $0.1,0.9$ \\
$N_{A M Y}$ & Number of neurons in Amygdala & 10 \\
$\theta$ & Threshold of conventional control & 5 \\
$\theta_{A M Y}$ & Threshold of emotional control & $0.0-1.0$ \\
$\theta_{R}$ & Threshold of Amygdala neurons & $0.3,0.6,0.9$ \\
$\theta^{c x}$ & Threshold of association cortex & 0.5 \\
$n_{R}$ & Threshold of Amygdala reward & $0.0-1.0$ \\
\hline
\end{tabular}

Table 2. Comparison of memory processing performance with results of mutual association simulations.

\begin{tabular}{ccc}
\hline Model & Storage time (steps) & Recollection time (steps) \\
\hline Conventional MCNN [7] [8] [9] & 18 & 17 \\
Amygdala - Hippocampus model & 8 & 35 \\
\hline
\end{tabular}




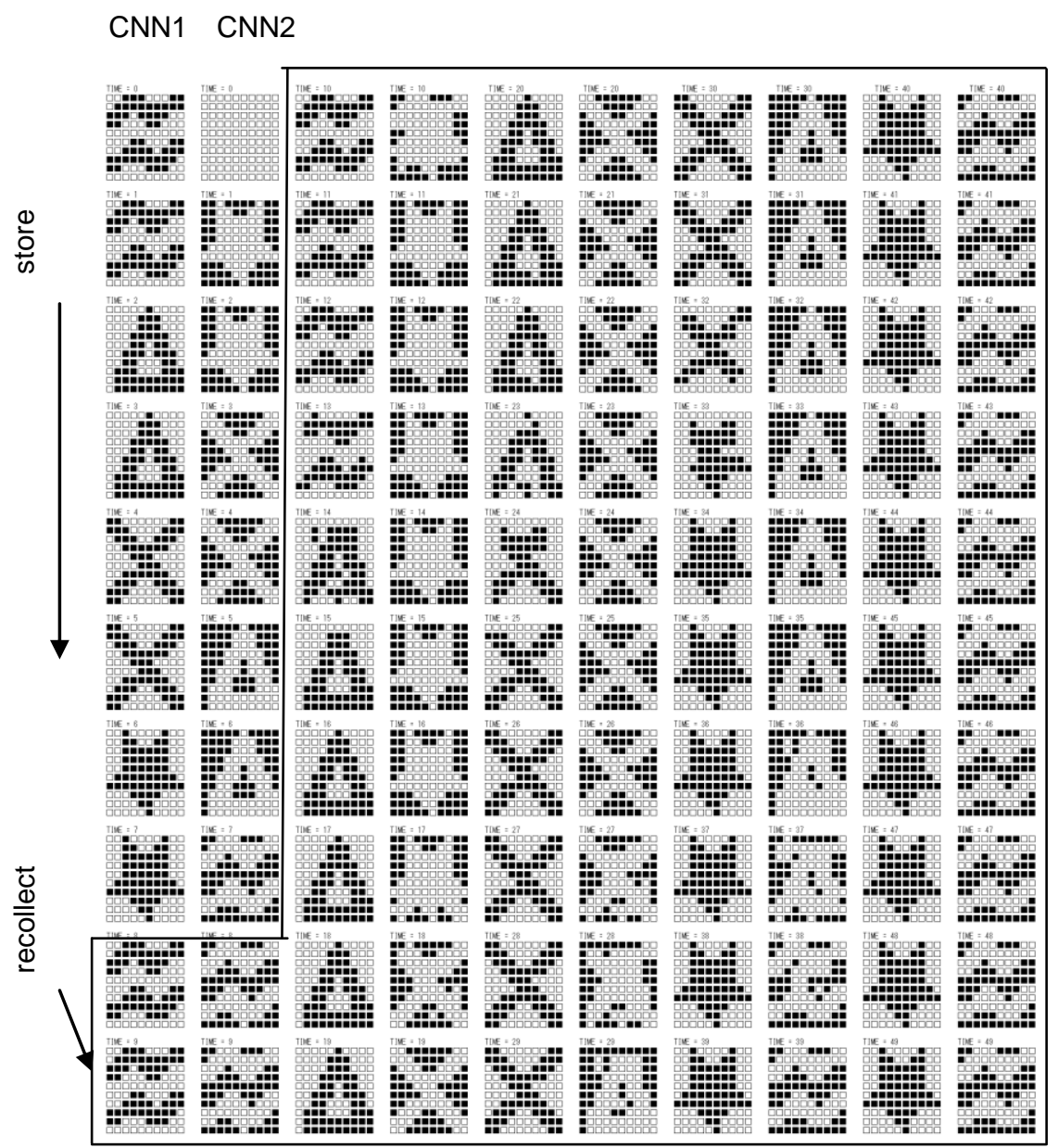

Fig. 2. Results of memory processing simulations with Amygadala - Hippocampus model proposed in [10].

Table 3. Comparison of successful rate of recollection with results of one-to-many times series pattern association simulations (\%).

\begin{tabular}{cccc}
\hline Models & Time series A & Time series B & Failed \\
\hline Hippocampus - neocortex model [8] [9] & 7 & 3 & 90 \\
Proposed model of limbic system & 9 & 9 & 82 \\
\hline
\end{tabular}

One simulation result of one-to-many association and long-term memory formation are shown in Fig. 3. One time series pattern was stored in CX2, while the rate of storage including failed one is reported by Table 3. The output of amygdala model during storage and recollection processes is shown in Fig. 4. Comparing with conventional hippocampus-neocortex model [8] [9], the model of limbic system proposed raised $8 \%$ rate of successful recollection. 


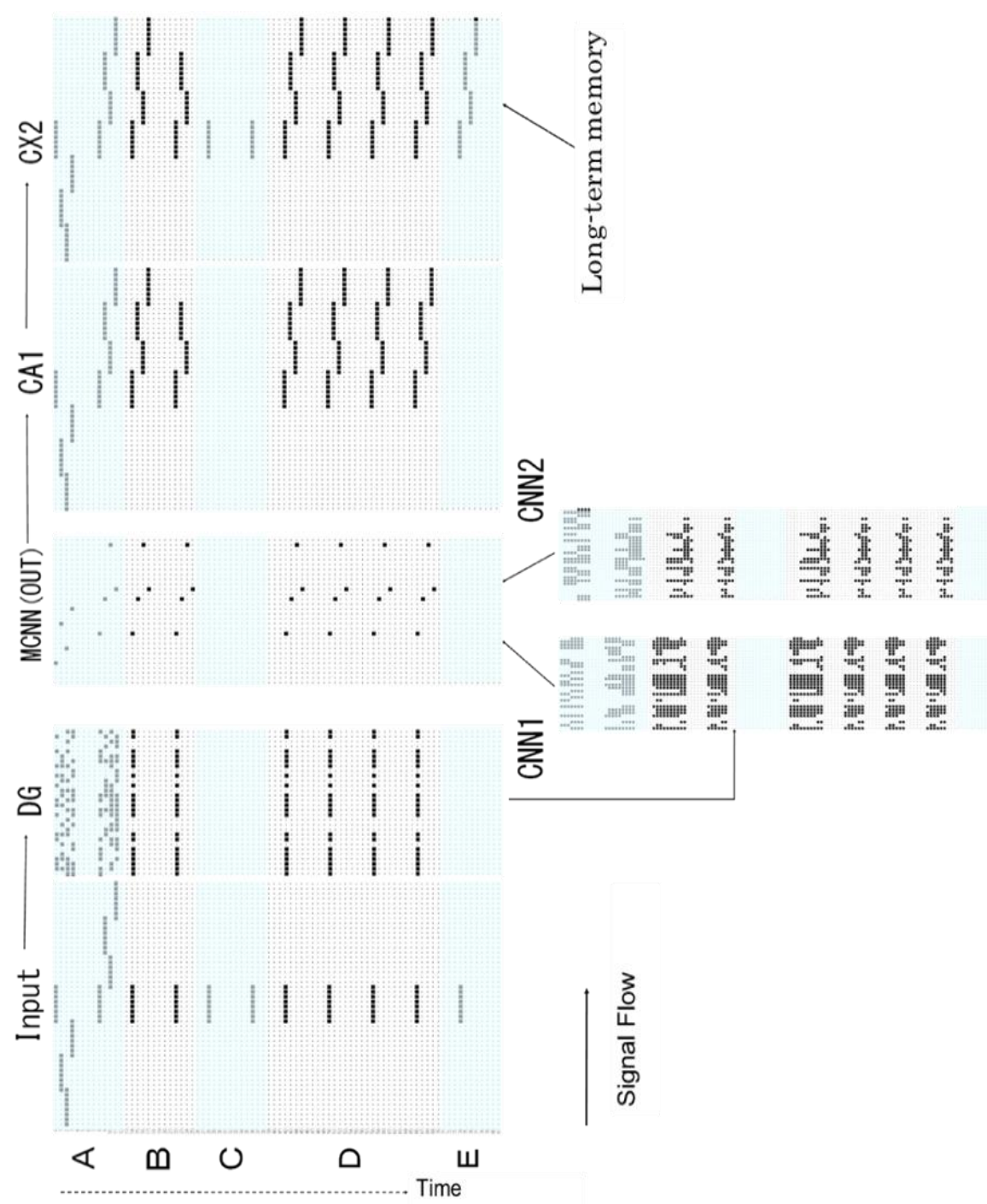

Fig. 3. Simulation results of long-term memory formation. Two time series patterns were presented during learning process and a piece of a common pattern was used to be a clue pattern until long-term memory was formed on CX2 layer.

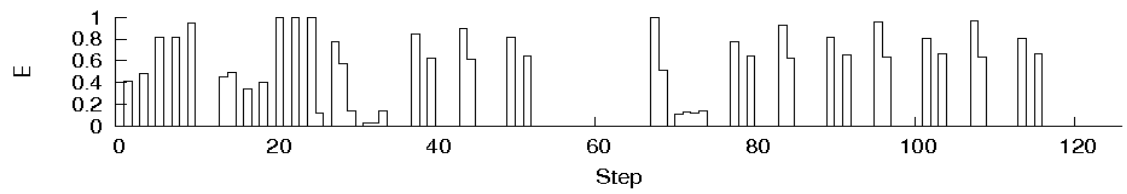

Fig. 4. The change of output of Amygdala during storage and recollection process. 


\section{Conclusion}

A model of limbic system is proposed by combining a conventional hippocampusneocortex model, chaotic neural works and an amygdala model. The proposed mathematical model can realize mutual association and long-term memory of multiple time series patterns with higher performance comparing with the conventional models. Different learning rules, such as Hebbian competition rule and reinforcement learning rule, are functionally adopted in the proposed model. This integration of memory models and emotion models gives an evidence of the realization probability of the computational artificial brain in the future.

\section{References}

1. Adachi, M., Aihara, K.: Associative Dynamics in Chaotic Neural Network. Neural Networks, No.10, pp. 83--98 (1997)

2. Aihara, K., Takabe, T., Toyoda, M.: Chaotic Neural Networks. Physics Letters A. Vo.144, No.6-7, pp. 333--340 (1990)

3. Balkenius, C., Morén, J.: Emotional Learning: A Computational Model the Amygdala, Cybernetics and Systems, Vol.32, No.6, pp. 611--636 (2000)

4. Ito, M., Kuroiwa, J., Sawada, Y., Miyake, S.: A model of hippocampus-neocortex for episodic memory. Proc. $5^{\text {th }}$ Intern. Conf. Neural Information Processing. 1P-16, pp. 431-434 (1998)

5. Ito, M., Miyake, S., Inawashiro, S., Kuroiwa, J., Sawada, Y.: Long term memory of temporal patterns in a hippocampus-cortex model. (in Japanese) Technical Report of IEICE. NLP2000, Vol.18, pp. 25--32 (2000)

6. Kajiwara, R., Takashima, I., Mimura, Y., Witter, M. P. Iijima, T.: Amygdala Input Promotes Spread of Excitatory Neural Activity from Perirhinal Cortex to the Entorhinal-Hippocampal circuit, J. europhysiology, Vol. 89, pp. 2176--2184 (2003)

7. Kuremoto, T., Eto, T., Kobayashi, K. and Obayashi, M.: A multilayered chaotic neural network for associative memory. Proc. of SICE Annual Conf., pp. 1020-1023 (2005)

8. Kuremoto, T., Eto, T., Kobayashi, K. and Obayashi, M.: A chaotic model of hippocampus neocortex. In: Wang, L., Chen, K., Ong, Y. S. (eds.), LNCS 3610, pp. 439--448 (2005)

9. Kuremoto, T., Eto, T., Kobayashi, K. and Obayashi, M.: A Hippocampus-Neocortex Model for Chaotic Association. In: Chen, K., Wang, L. (eds.) Trends in Neural Computation (Studies in Computational Intelligence Vol.35), pp. 111--133 (2006)

10. Kuremoto, T., Ohta, T., Obayashi, M., Kobayashi, K.: A Dynamic Associative System by Adopting an Amygdala Model. Artif. Life and Robotics, Vol. 13, No.2, pp.478--482 (2009)

11. MaGaugh, J., L., Cahill, L., Roozendaal, B.: Involvement of the Amygdala in Memory Storage: Interation with Other Brain Systems. Proc. Natl. Acad. Sci. USA, Vol. 93, pp. 13508-13514 (1996)

12. Mizutani, S., Sano, T. Uchiyama, T., Sonehara, N.: Controlling Chaos in Chaotic Neural Networks. Electronics and Communications in Japan, Part III: Fundamental Electronics Science, Vol. 81, No.8, pp. 73--82 (1998)

13. Morén, J., Balkenius, C.: A Computational Model of Emotional Learning in the Amygdala. Proc. of the 6th Intern. Conf. on the Simulation of Adaptive Behavior, Cambridge MA, MIT Press (2000)

14. Rouhani, H., Jalili, M., Araabi, B. N., Eppler, W., Luscas, W.: Brain Emotional Learning Based Intelligent Controller Applied to Neurofuzzy Model of Micro-Heat Exchanger. Expert Sys. with Appli., Vol. 32, pp. 911--918 (2007) 